\title{
A REMARK ON FINITELY GENERATED NILPOTENT GROUPS
}

\section{GRAHAM HIGMAN}

In this note we use the word nilpotent in the strong sense; a group $G$ is nilpotent if its lower central series, defined by $H_{0}=G$ and $H_{i+1}$ $=\left(H_{i}, G\right)$, terminates in the identity in a finite number $c$ of steps, and $c$ is then the class of $G$. As usual, $G^{n}$ denotes the subgroup of $G$ generated by the $n$th powers of elements of $G$. Our object is to prove the following result.

THEOREM 1. If $G$ is a finitely generated nilpotent group, then the intersection of the groups $G^{p}$, for any infinite set of primes $p$, is finite.

We recall first some well known facts about finitely generated nilpotent groups. They will be found, in essence, in Hall [2] and Hirsch [3]. First, if $X$ is a subset of $G$ that generates $G$ modulo its derived group $H_{1}$, then $H_{i-1}$ is generated modulo $H_{i}$ by the left normed commutators

$$
\left(\cdots\left(\left(x_{1}, x_{2}\right), x_{3}\right), \cdots, x_{i}\right), \quad x_{r} \in X .
$$

Thus $H_{i-1}$ is finitely generated modulo $H_{i}$. In particular, $H_{c-1}$ is a finitely generated abelian group, and its subgroups therefore satisfy the maximal condition. By induction on $c$, so do those of $G$. Again, if any of $x_{r}$ is of finite order modulo $H_{1}$, then the commutator (1) is of finite order modulo $H_{i}$. Hence finitely many elements of $G$ of finite order generate a finite subgroup, and it follows from the maximal condition on the subgroups of $G$ that in fact there are only a finite number of elements of finite order in $G$. In particular, there are only a finite number of primes $p$ for which $G$ contains elements of order $p$. Lastly, for a prime $q$ greater than the class $c$ of $G$, Hall's formula becomes

$$
(x y)^{q}=x^{q} y^{q} z_{1}^{q} \cdots z_{r}^{q}
$$

where each $z_{j}$ is a commutator in $x$ and $y$. It follows easily, by backward induction on the weight of $y$ (the least integer $i$ such that $y$ does not belong to $H_{i}$ ), that for some $u$ in $G, x^{q} y^{q}=u^{q}$. That is, $G^{q}$ is not merely generated by, but consists of the $q$ th powers of elements of $G$.

We can now prove Theorem 1 . If the class $c$ of $G$ is 1 , so that $G$ is abelian, the theorem follows immediately from the basis theorem for finitely generated abelian groups. We therefore use induction on $c$, and

Received by the editors June 3, 1954. 
assume $c>1$. Let $K$ be the intersection of an infinite set of subgroups $G^{p}$. By the hypothesis of the induction, $K H_{c-1} / H_{c-1}$ is finite, and hence so is the isomorphic group $K /\left(K \cap H_{c-1}\right)$. It is sufficient therefore to prove that $K \cap H_{c-1}$ is finite. If $y \in K$, then surely $y \in G^{q}$ for an infinity of primes $q$ with $q>c$; thus for an infinity of $q$ there exists $y_{q}$ such that $y_{q}^{q}=y$. If also $y \in H_{c-1}$, the cyclic group $\{y\}$ is normal, and unless $y_{q} \in\{y\}$, the factor group $G /\{y\}$ contains an element of order $q$. Since this is possible for only a finite set of primes $q$, for some $q, y_{q} \in\{y\}$, whence $y$ is of finite order. That is, $K \cap H_{c-1}$ contains only elements of finite order, and is therefore a finite group. This concludes the proof.

It is perhaps of interest to remark that Theorem 1 yields a short proof of the following theorem of Baer [1].

Theorem 2. There is an integer $n$ such that the intersection of all characteristic subgroups of $G$ whose indices are prime powers $p^{a}$ with $a \leqq n$ is the identity.

For, by a theorem of Hirsch [4], $G$ (or, indeed, any soluble group with the maximal condition for subgroups) has a subgroup $N$ of finite index which contains no elements of finite order. We can take this subgroup to be characteristic. For, if its index is $h$, we can replace it by the intersection of all subgroups of $G$ of index $h$, which (Baer, loc. cit.) is still of finite index. Then $G / N$ is a finite nilpotent group, and so the direct product of its Sylow subgroups, whence $N$ is the intersection of a finite set of characteristic subgroups of $G$, whose indices are prime powers. If to these we add any infinite set of subgroups $G^{p}$, we obtain a set whose intersection is the identity. For this intersection contains no element of finite order by choice of $N$, and none of infinite order by Theorem 1. All the groups are characteristic subgroups of $G$ of prime power index $p^{a}$, and to show that the exponents $a$ are bounded, we may concentrate on the groups $G^{p}$, since the others are a finite set only. But if the number of generators of $H_{i-1} / H_{i}$ is $r(i)$, the index of $G^{p}$ is $p^{a}$ with $a \leqq r(1)+r(2)+\cdots$ $\operatorname{tr}(c)$.

\section{REFERENCES}

1. Reinhold Baer, Das Hyperzentrum einer Gruppe III, Math. Zeit. vol. 59 (1953) pp. 299-338.

2. P. Hall, $A$ contribution to the theory of groups of prime power order, Proc. London Math. Soc. (2) vol. 36 (1933) pp. 29-95.

3. K. A. Hirsch, On infinite soluble groups II, ibid. vol. 44 (1938) pp. 336-344.

4. - On infinite soluble groups III, ibid. vol. 49 (1945-47) pp. 184-194.

The University, Manchester, England 